\title{
Fazit zu den wichtigsten Steuerarten
}

\begin{abstract}
Das deutsche Steuerwesen zeichnet sich durch eine hohe Komplexität aus. Dazu trägt die Vielzahl der Steuerarten bei. Allerdings ist auch die Berechnung der Steuerhöhe in der Regel alles andere als trivial. Lernen Sie, das Steuerrecht kritisch zu beurteilen und bilden Sie sich eine eigene Meinung.
\end{abstract}

Die Ausführungen zeigen die hohe Komplexität der einzelnen Steuerarten, die für Unternehmen und Belegschaft eine große Rolle spielen. Gerade die in der Berechnung eher einfache Umsatzsteuer wirft zahlreiche Fragen auf, die sich durch die Begrifflichkeiten ergeben.

Ob die Höhe der Steuerlast berechtigt ist, kann nicht abschließend gesagt werden. Dazu müssten die Leistungen gegenüber gestellt werden, die letztlich mithilfe der Steuereinnahmen finanziert werden. Auf jeden Fall gibt es hier zwischen den Ländern der Europäischen Union und der gesamten Welt große Unterschiede.

Grundsätzlich besteht der Verdacht, dass aufgrund der Bemühungen um eine gerechte Steuergesetzgebung so viele Details und Besonderheiten in die Steuergesetze eingearbeitet sind, dass nur noch absolute Spezialisten die Korrektheit einer Steuererklärung gewährleisten können. Dies führt dann aber letztlich zu weniger Steuergerechtigkeit, da eine Vielzahl der Betroffenen ihre eigenen Rechte und Pflichten kaum verstehen kann.

Für alle Unternehmen empfiehlt sich der Gang zum Steuerberater. Aber auch Arbeitnehmer, die mehr Einkünfte erzielen als nur solche aus unselbstständiger Arbeit, sind mit einer Fachberatung auf dem richtigen Weg. Bedenken Sie hier nur die vielen Eigentümer von Fotovoltaik-Anlagen auf den Dächern ihrer Wohnhäuser, die Strom ins öffentliche Stromnetz einspeisen und damit zum Gewerbetreibenden (und häufig auch freiwillig zum Umsatzsteuerpflichtigen) werden. 